\title{
Relation between Magnetostriction and Magnetic Domains in Changing Direction of Grain Oriented Silicon Steel Sheet
}

\author{
Hiroaki MASUI, Yukio MATSUO, ${ }^{1)}$ Masato MIZOKAMI and Hisashi MOGI') \\ Electromagnetic Materials, Steel Research Laboratory, Nippon Steel Corporation, Shintomi, Futtsu, Chiba-ken, 293 Japan. \\ 1) Yawata Laboratory, Nippon Steel Corporation, Tobihata, Tobata-ku, Kitakyushu, Fukuoka-ken, 804 Japan.
}

(Received on May 9, 1995; accepted in final form on July 24, 1995)

\begin{abstract}
Research has been carried out on the magnetostriction measured in changing direction of grain oriented silicon steel sheets. When the magnetic field is applied in a direction of $45^{\circ}$ off the rolling direction normally parallel to Goss texture orientation, the magnetostriction indicated negative value regardless of compressive stress applied to specimen and showed favourably smaller negative value in poor coating stress condition rather than in good one, which had not been easily imagined by the experience concerning to the magnetostriction in the rolling direction. This was explained by a fact that the $90^{\circ}$ supplementary domains by compressive applied stress followed by the strain elastic energy theory, which cause positive magnetostriction when the specimen is exposed to magnetic field, is compensated by negative magnetostriction by the abnormal supplementary domains newly generated under magnetic field. The abnormal supplementary domain occurs in specimen with the angle to the rolling direction of about $30^{\circ}-60^{\circ}$, which was tried to explain by magnetic domain energy. When the magnetic field is applied in a direction of $90^{\circ}$ off the rolling direction, the magnetostriction indicated the largest positive value which had been easily supposed by the reaction of the $180^{\circ}$ main domain exposed to magnetic field transversely.
\end{abstract}

KEY WORDS: magnetostriction; strain elastic energy; grain oriented silicon steel; easy magnetization axis; compressive stress; the abnormal supplementary domain.

\section{Introduction}

Recently noise generated by transformer has emerged as important from an environmental point of view and also in relation to the efficiency of energy consumption. One of the most important properties of grain oriented silicon steel for the transformer is the magnetostriction which causes noise evidently when the transformer is in operation.

Nowadays, as well as the magnetostriction of the steel sheet cut in the rolling direction, ${ }^{1-6)}$ which is main for the use of large parts such as limb and yoke of the transformer, magnetostriction of the sheets cut $45^{\circ}$ or $90^{\circ}$ off the rolling direction is collecting remarkable attention. The background is that noise at small portion in the joint corners between limb and yoke, through which the magnetic flux direction deflects considerably off the rolling direction in the steel sheet, can be hardly neglected. ${ }^{7-9)}$

It is already known ${ }^{10-13)}$ that the magnetostriction of the silicon steel is caused by movement of the $90^{\circ}$ supplementary domain walls. When the supplementary domains are generated by applied stress, the steel shows positive magnetostriction when the specimen is exposed to magnetic field. ${ }^{1,2)}$ It is also known that there is a close relation between the formation of magnetic domains and elastic stress to the steel in such a manner that the domains tend to form in $\langle 100\rangle$ direction of the silicon steel if tensile stress is applied along the $\langle 100\rangle$ easy magnetization axis. ${ }^{12-15)}$ The elastic stress brings about strain elastic energy in the axis. This means that the formation of the supplementary domains, which cause large magnetostriction when the steel is exposed to magnetic field, may be presumably dominated by a relation of strain elastic energy in easy magnetization axes $(y[010]$ and $z[001])$ with transverse direction to the rolling direction normally parallel to the main $180^{\circ}$ domains, to that in $x[100]$ easy magnetization axis. ${ }^{1)}$ In a previous research report, ${ }^{1)}$ it was made clear that the deteriorating influence of stress on the magnetostriction in the rolling direction of grain oriented silicon steel is calculated by means of the formulae considering a formation of the $90^{\circ}$ supplementary domains based on a generalized condition that the supplementary domains are formed when the strain elastic energy in an easy magnetization axis $x[100]$, which is generally parallel to the rolling direction in the Goss orientation texture, is smaller than that in $y[010]$ and $z$ [001] (other easy magnetization axes) under e.g. compressive stress applied to the specimen. From both industrial point of view and physical one, it should have been investigated whether the strain elastic energy theory may be applied to the formation of the supplementary domains even for the specimens of $45^{\circ}$ or $90^{\circ}$ off the rolling direction without magnetic field and whether it may be possible to predict the magnetostriction of them. 
This study aims to, first of all, ascertain the strain elastic energy theory on the formation of the $90^{\circ}$ supplementary domains without magnetic field in grain oriented silicon steel sheets even cut with angles from $0^{\circ}$ to $90^{\circ}$ to the rolling direction and, consequently, find a mechanism of the magnetostriction in the magnetized state in changing direction of the steel sheets.

\section{Experimental Procedure}

Grain oriented $3 \%$-silicon steel (HI-B, $0.23 \mathrm{~mm}$, B8 : 1.92T) was prepared for experiments. Two kinds of surface coating stress conditions were made in such a way as one (sample A) with high tension insulation coating film on mill glass and another (sample B) by dissolving off the high tension coating film from mill glass by alkali solution. Table 1 shows magnetic properties of the samples. In order to investigate the magnetostriction as well as a behavior of the magnetic domains in changing direction of the grain oriented silicon steel sheet, specimens were cut with various angles from the rolling direction. Magnetostriction specimens of $60 \times 300 \mathrm{~mm}$ were taken from the rolling direction, and $45^{\circ}$ and $90^{\circ}$ direction from the rolling direction. Specimens of $15 \times 70 \mathrm{~mm}$ for the observation of magnetic domains by scanning electron microscope, were taken from the rolling direction and from every $15^{\circ}$ angle between $0^{\circ}$ and $90^{\circ}$ from the rolling direction. Schematic drawings for these are depicted in Fig. 1 and definition of the angles are $\chi$ for the magnetostriction specimen and $\phi$ for the scanning electron microscope specimen, respectively. The $180^{\circ}$ main domains are not always perfectly parallel to the rolling direction. Figure 1 shows, therefore, a definition of angle between direction of the $180^{\circ}$ main domains and direction of magnetic field (magnetization) in the scanning electron microscopic observation, as $\theta$. It is noted that the direction of applied stress as well as magnetic field is always parallel to longitudinal direction of the specimens for the scanning electron microscope as well as for the magnetostriction. The definition of angle between applied stress and $x[100]$ easy magnetization axis is needed to calculate the strain elastic energy in this study and this study can use sign of $\alpha$ which was already defined in previous report ${ }^{1)}$ as described in Fig. 13.

Magnetostriction was measured in a range of $0.5-1.3 \mathrm{~T}$ at $50 \mathrm{~Hz}$ by using a Doppler type magnetostriction tester simultaneously applying compressive stress from 0 to $3 \mathrm{MPa}$ along longitudinal direction. Observation of magnetic domains both without any compulsory magnetic field and with magnetic field( A.C., D.C.), was done by a $200 \mathrm{kV}$ scanning electron microscope enclosing special device, which may apply compressive stress of 0 to $10 \mathrm{MPa}$ to the longitudinal direction of the specimens. Coating stress was measured by stripping off mill glass, and the coating film as well in sample A, only on one side of the specimen, combined with the aid of checking the coefficient of elasticity by precise tensile tester. Some specimens were scratched by using a ball-point pen with a pitch of $10 \mathrm{~mm}$ distance perpendicular to the longitudinal direction with about $40 \mu \mathrm{m}$ contact width on the steel surface, which is frequently convenient to recognize dimension and direction of magnetic domains.

\section{Experimental Results}

\subsection{Magnetostriction}

Two kinds of the different surface coating stress samples were prepared. Sample A had a coating stress of 9.0 MPa,while sample B showed 2.0 MPa. Magnetostriction (0-Peak, a difference from magnetostriction at maximum flux density $T_{\max }$ to that at 0 flux density $T_{0}$ )

Table 1. Magnetic properties of the samples.

\begin{tabular}{|c|c|c|c|c|c|c|}
\hline \multirow{2}{*}{ Sample } & \multirow{2}{*}{$\begin{array}{l}\text { Thickness } \\
(\mathrm{mm})\end{array}$} & \multirow{2}{*}{$\begin{array}{l}\text { Coating stress } \\
(\mathrm{MPa})\end{array}$} & \multicolumn{3}{|c|}{ Iron loss $(W / \mathrm{kg})$} & \multirow{2}{*}{$\begin{array}{l}\text { Magnetic induction } \\
(800 \mathrm{~A} / \mathrm{m} \text {, Tesla) }\end{array}$} \\
\hline & & & $W_{13 / 50}$ & $W_{15 / 50}$ & $W_{17 / 50}$ & \\
\hline A & 0.23 & 9.0 & 0.45 & 0.61 & 0.85 & 1.925 \\
\hline B & 0.23 & 2.0 & 0.55 & 0.72 & 1.02 & 1.930 \\
\hline
\end{tabular}

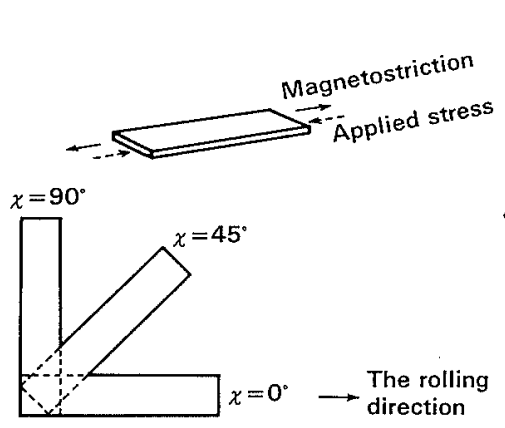

(a)

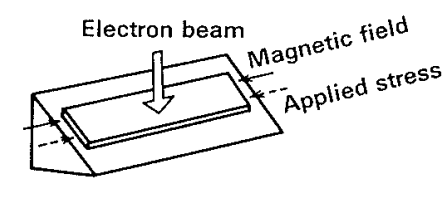

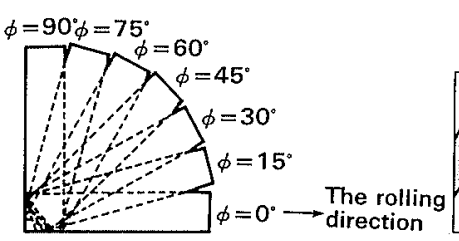

(b)

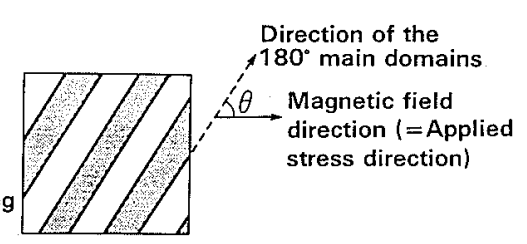

(c)

Fig. 1. Schematic experimental procedures and definition of the angles.

(a) Magnetostriction; $\chi$ : Angle between the magnetostriction specimen and the rolling direction

(b) Observation of magnetic domains by scanning electron microscope; $\phi$ : Angle between the scanning electron microscope specimen and the rolling direction

(c) 0 : Angle between the $180^{\circ}$ main domains and magnetic field. 
at $1.3 \mathrm{~T}$ in the specimens with the angle $\chi$ (angle between the magnetostriction specimen and the rolling direction $=0^{\circ}, 45^{\circ}$ and $90^{\circ}$ from the rolling direction is shown in Fig. 2. In the test of the $\chi=0^{\circ}$ samples, as same as previously reported, ${ }^{1)}$ positive elongation became remarkably large in poor coating stress sample B even with small increase of compressive applied stress, while extremely small increase of elongation was perceived even on $3 \mathrm{MPa}$ compressive applied stress in sample A. On the other hand, in the test of the $\chi=45^{\circ}$ samples, the magnetostriction showed, contrary to an expectation, negative value regardless of compressive applied stress on both sample A and sample B. Further interesting is that sample B, poor coating stress sample, had rather favourably smaller negative magnetostriction value than sample $\mathrm{A}$. In the test of the $\chi=90^{\circ}$ samples, it indicated the largest positive elongation on both samples. Butterfly loops for the magnetostriction are shown in Fig. 3(a) $\left(\chi=0^{\circ}\right)$, Fig. 3(b) $\left(\chi=45^{\circ}\right)$ and Fig. 3(c) $\left(\chi=90^{\circ}\right)$.

\subsection{Observation of Magnetic Domains by Scanning Electron Microscope}

Observation of magnetic domains by scanning electron microscope was carried out in several conditions: demagnetized state with and without compressive applied stress; and magnetized state with and without compressive applied stress. It should be noted that micrographs of domain structure taken by scanning electron microscope should be corrected with the standard angle scale chart in Fig. 4. The distortion was caused by the difference in magnitude for $x$ and $y$ directions which is easily supposed by Fig. 1(b) and angle $\theta$ (angle between the $180^{\circ}$ main domains and magnetic field) was thereby corrected hereafter which were

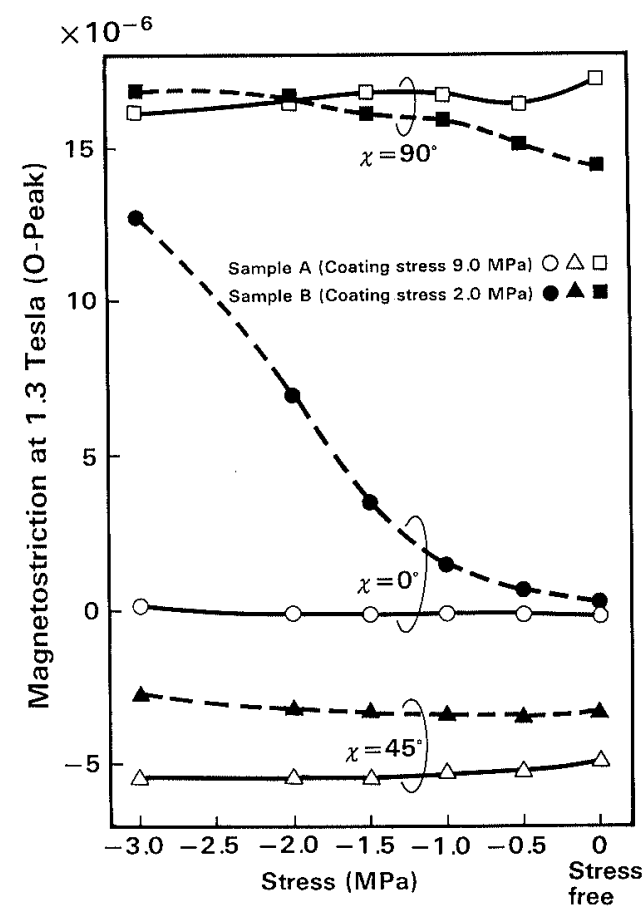

Fig. 2. Influence of $\chi$ (angle between the magnetostriction specimen and the rolling direction) and applied compressive stress on magnetostriction (1.3 Tesla, $50 \mathrm{~Hz}, 0$-Peak) in different coating stress sample A and B.

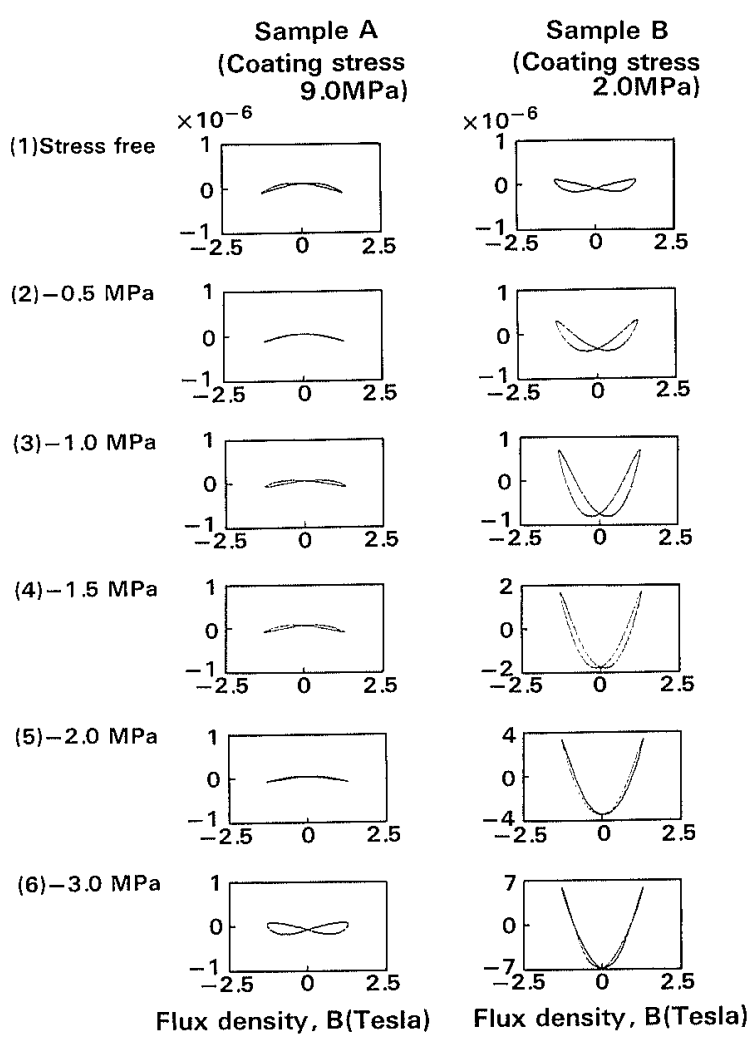

Fig. 3(a). Butterfly loops of magnetostriction at 1.3 Tesla $(50 \mathrm{~Hz})$ under stress free and compressive stress conditions in different coating stress samples with $\chi$ (angle between the magnetostriction specimen and the rolling direction $)=0^{\circ}$.

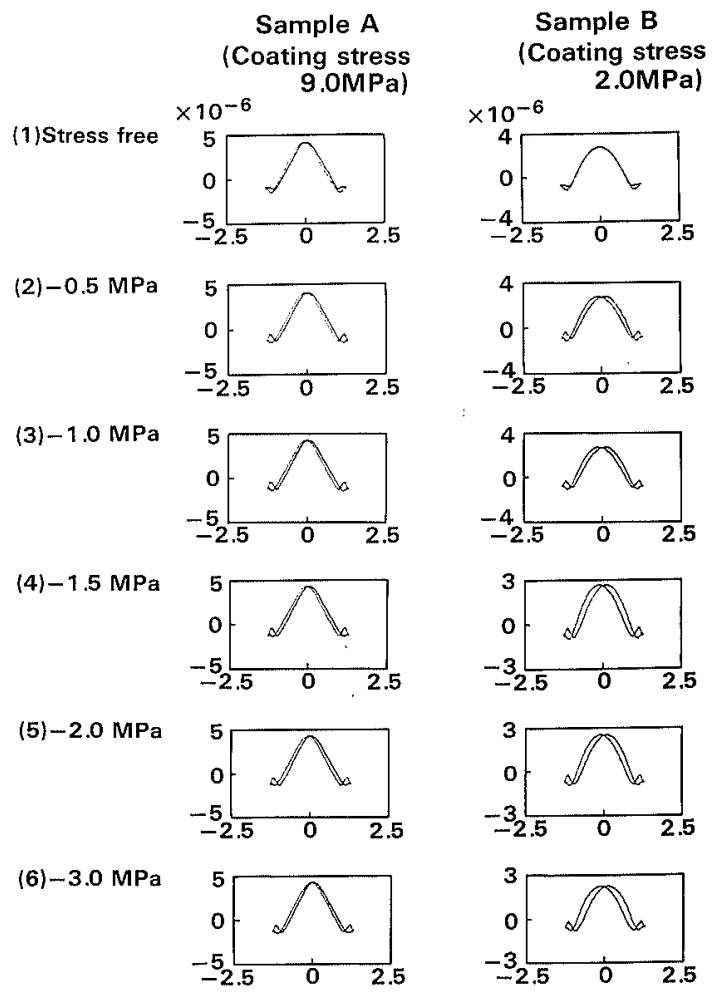

Flux density, B(Tesla) Flux density, B(Tesla)

Fig. 3(b). Butterfly loops of magnetostriction at 1.3 Tesla $(50 \mathrm{~Hz})$ under stress free and compressive stress conditions in different coating stress samples with $\chi$ (angle between the magnetostriction specimen and the rolling direction $)=45^{\circ}$. 

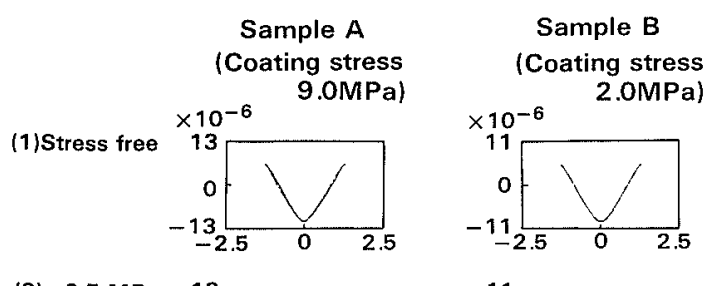

(2) $-0.5 \mathrm{MPa}$
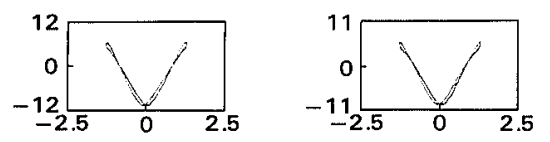

(3) $-1.0 \mathrm{MPa}$
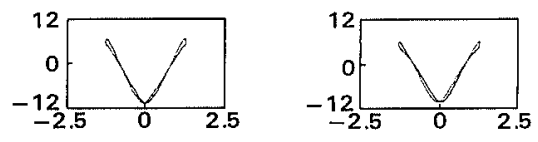

(4) $-1.5 \mathrm{MPa}$
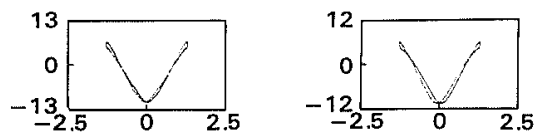

(5) $-2.0 \mathrm{MPa}$
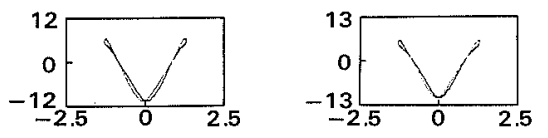

(6) $-3.0 \mathrm{MPa}$
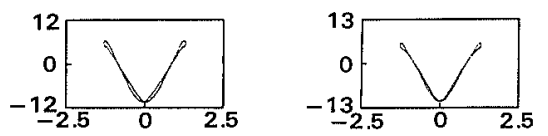

Flux density, B(Tesla) Flux density, B(Tesla)

Fig. 3(c). Butterfly loops of magnetostriction at 1.3 Tesla $(50 \mathrm{~Hz})$ under stress free and compressive stress conditions in different coating stress samples with $\chi$ (angle between the magnetostriction specimen and the rolling direction) $=90^{\circ}$.

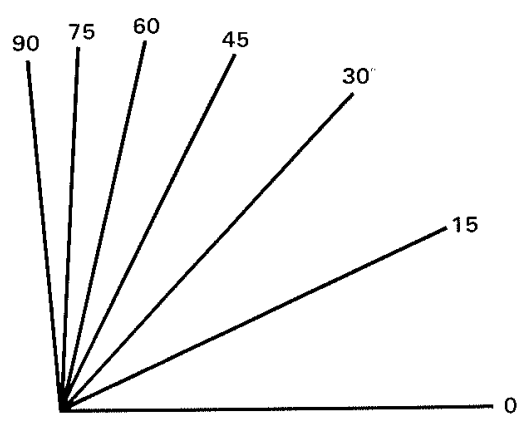

Fig. 4. Correction chart of angle for observed magnetic domains in the $200 \mathrm{kV}$ scanning electron microscope.

described in each micrograph. Figure 5 shows magnetic domains of the $\phi$ (angle between the scanning electron microscope specimen and the rolling direction $)=0^{\circ}$ sample $\mathrm{B}$ (coating stress: $2.0 \mathrm{MPa}$ ) with $\theta$ (angle between the $180^{\circ}$ main domains and magnetic field) $=0^{\circ}$ exhibiting the $90^{\circ}$ supplementary domains by compressive applied stress and the $180^{\circ}$ main domain wall displacement under magnetic field, which are most familiar in grain oriented silicon steel. Figure 6 shows magnetic domains of the $\phi=0^{\circ}$ sample A (coating stress: $9.0 \mathrm{MPa}$ ) with partly $\theta=0^{\circ}$ and partly $\theta=12^{\circ}$, exhibiting also same phenomenon as in Fig. 5. Figure 7 shows magnetic domains of the $\phi=45^{\circ}$ sample $A$ with $\theta=38-44^{\circ}$ indicating a few supplementary domains by compressive applied stress and newly generated abnormal supplementary domains under magnetic field which seems to occur independently of the supplementary domains by the compressive ap- plied stress. Figure $\mathbf{8}$ shows magnetic domains of the $\phi=45^{\circ}$ sample $\mathrm{B}$ with $\theta=45^{\circ}$ exhibiting still easy occurrence of the supplementary domains under compressive applied stress due to poor coating stress, together with the abnormal supplementary domains under magnetic field. Figure 9 shows magnetic domains of the $\phi=60^{\circ}$ sample A with $\theta=52^{\circ}$ indicating that there seems no supplementary domains by applied compressive stress, while there are still the abnormal supplementary domains under magnetic field. Figure 10 shows magnetic domains of the $\phi=75^{\circ}$ sample A with $\theta=68^{\circ}$ exhibiting that there scarcely appear the abnormal supplementary domains under magnetic field. Finally, Fig. 11 shows magnetic domains of the $\phi=90^{\circ}$ sample A with $\theta=88^{\circ}$ exhibiting the representative singular domains in the specimen when the $180^{\circ}$ main domains are exposed to magnetic field transverse to the rolling direction. In largest $\theta$ as in Fig. 11 , it is necessary to elevate a voltage for electron beam to take high contrast scanning electron micrographs of magnetic domains. Thus, it was made clear that there are two kinds of supplementary domains, one of which occurs by applied stress and the other is generated under magnetic field only in the samples with considerable amount of degree in angle off the rolling direction.

It seems clear that there is an occurrence range in $\theta$ around about $\theta=45^{\circ}$ on the appearance of the abnormal supplementary domains under magnetic field. Figure 12 shows a map with a bar indicating which of the $180^{\circ}$ main domain wall displacement or the abnormal supplementary domain appearance occurred mainly or subsidiarily under magnetic field in relation to the angle $\theta$ (angle between the $180^{\circ}$ main domains and magnetic field) by the scanning electron micrographs.

\section{Discussion}

As described in 1 (Introduction), it was made clear ${ }^{1)}$ that a formation of the $90^{\circ}$ supplementary domains considers the formulae based on a generalized condition that the supplementary domains are formed when the strain elastic energy in an easy magnetization axis $x$ [100], which is normally parallel to the rolling direction in the Gross orientation texture, is smaller than that in $y[010]$ and $z$ [001] (other easy magnetization axes) under stresses applied to the specimen in relation to coating stress even such a sophisticated case as under bi-axial stresses. When the strain elastic energies in easy magnetization axes $x[100], y[010]$ and $z$ [001] are defined as $E_{x[100]}^{\mathrm{e} 1}, E_{y[010]}^{\mathrm{el}}$ and $E_{z[001]}^{\mathrm{el}}$, a generalized condition that the $90^{\circ}$ supplementary domains are formed, is expressed as $E_{x[100]}^{\mathrm{el}} \leqq E_{y[010](z[001]) \cdot}^{\mathrm{el}}{ }^{1)}$ The strain elastic energy e.g. $E_{x[100]}^{\mathrm{e}]}$ in the easy magnetization axis [100] is, however, induced not only simply by a stress in the rolling direction, but also by a combination with other stresses including coating stress. ${ }^{1)}$ The calculation was carried out in accordance with mathematical process, given e.g. by Masui, ${ }^{1,22)}$ and the equations are referred to in Table 2 with definition of angles denoted in Fig. 13. In Table 2, the coordinates $X Y Z$ are those of the specimen, where $X$ is axis of longitudinal direction of specimen and $Y$ is width direction as well as $Z$ is 


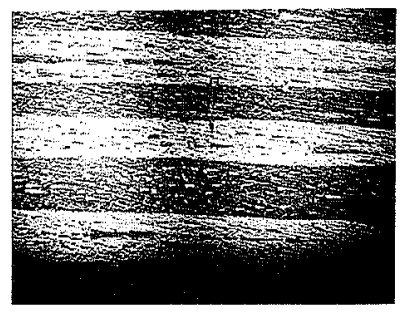

(a) Stress free, Demagnetized

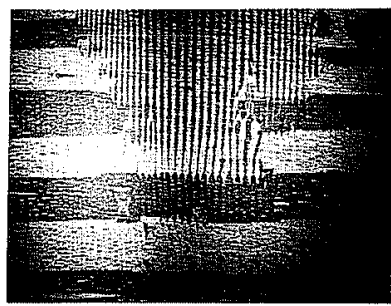

(c) $1 \mathrm{MPa}$ Compressive stress, Demagnetized

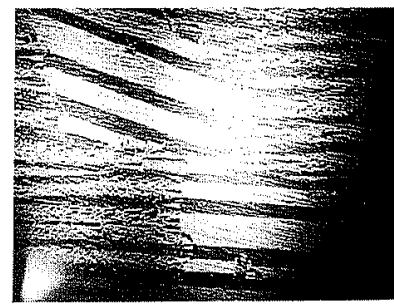

(a) Stress free, Demagnetized

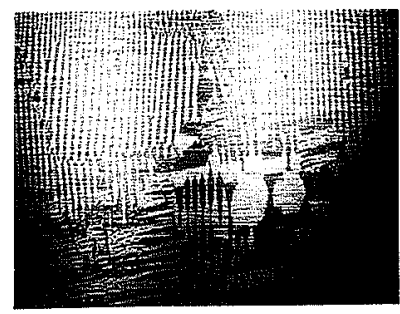

(c) $2.5 \mathrm{MPa}$ Compressive stress, Demagnetized

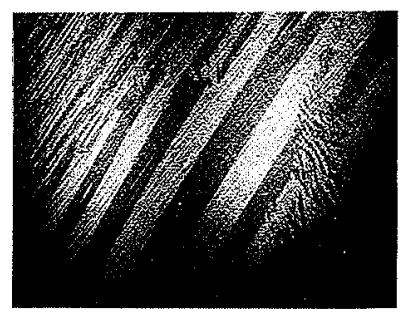

(a) $4 \mathrm{MPa}$ Compressive stress, Demagnetized

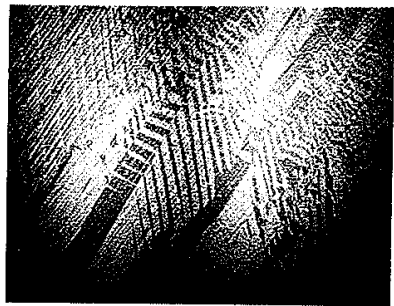

(c) $4 \mathrm{MPa}$ Compressive stress, Magnetized(D.C.) $B=9 K G$

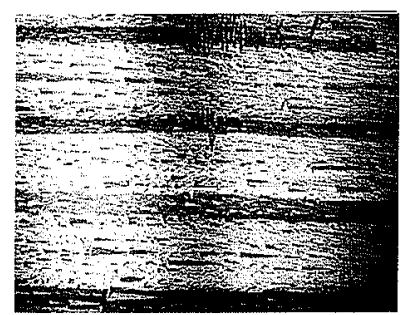

(b) Stress free, Magnetized(D.C.) $\mathrm{B}=12 \mathrm{KG}$

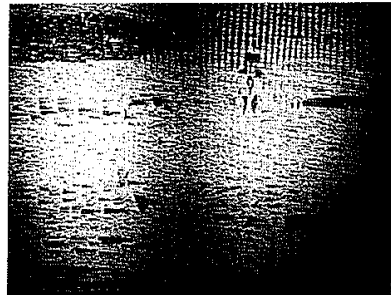

(d) $1 \mathrm{MPa}$ Compressive stress, Magnetized(D.C.) $B=12 \mathrm{KG}$

$\overline{1 \mathrm{~mm}}$

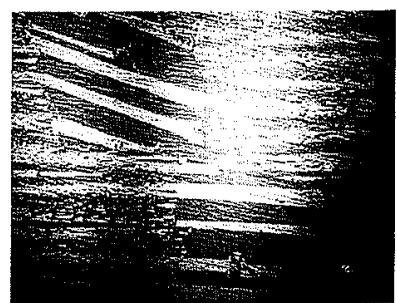

(b) Stress free, Magnetized(D.C.) $\mathrm{B}=12 \mathrm{KG}$

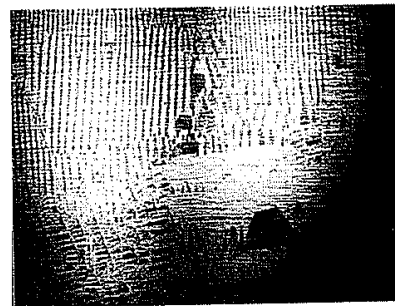

(d) $2.5 \mathrm{MPa}$ Compressive stress, Magnetized(D.C.) $B=12 \mathrm{KG}$

$\stackrel{1 \mathrm{~mm}}{ }$

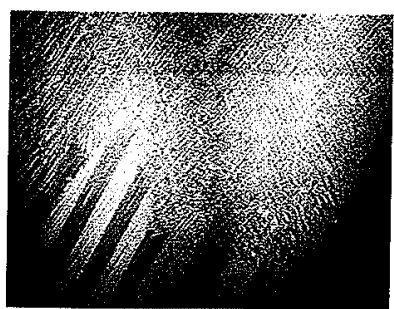

(b) $4 \mathrm{MPa}$ Compressive stress, Magnetized(A.C.) $B=9 K G$

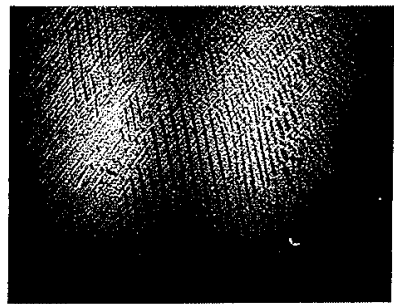

(d) $4 \mathrm{MPa}$ Compressive stress, Magnetized(D.C.) $B=11 \mathrm{KG}$
Fig. 5.

Magnetic domain structures in $\phi$ (angle between the scanning electron microscope specimen and the rolling direction) $=0^{\circ}$ sample $B$ (coating stress $2.0 \mathrm{MPa}$ ) with $\theta$ (angle between the $180^{\circ}$ main domains and magnetic field $)=0^{\circ}$ under various stress and magnetic field conditions.
Fig. 6.

Magnetic domain structures in $\phi=0^{\circ}$ sample A (coating stress $9.0 \mathrm{MPa}$ ) with $\theta=0^{\circ}, 12^{\circ}$ under various stress and mag. netic field conditions.
Fig. 7.

Magnetic domain structures in $\phi=45^{\circ}$ sample A with $\theta=38-44^{\circ}$ under various stress and magnetic field conditions. 


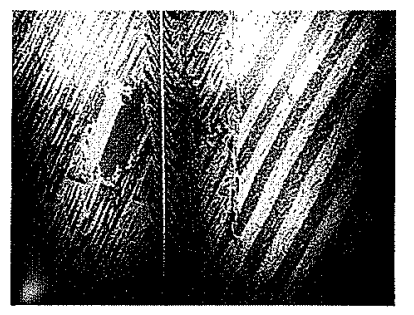

(a) Stress free, Demagnetized

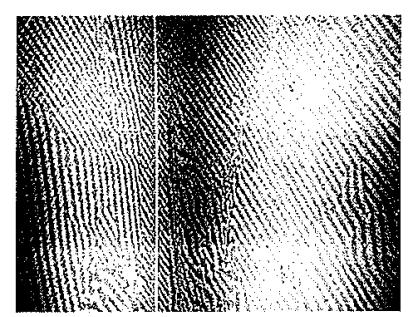

(c) $2.5 \mathrm{MPa}$ Compressive stress, Demagnetized

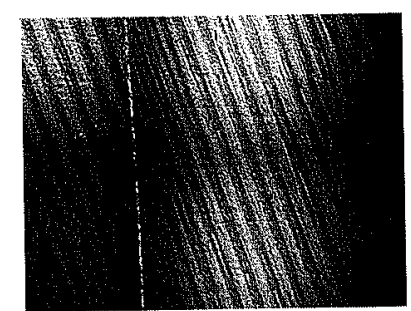

(a) Stress free, Demagnetized

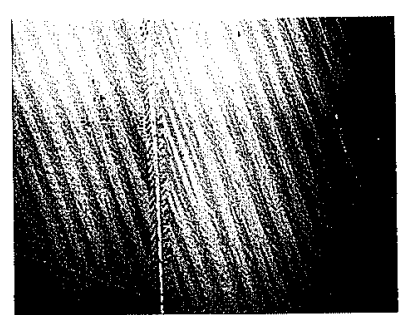

(c) $10 \mathrm{MPa}$ Compressive stress, Demagnetized

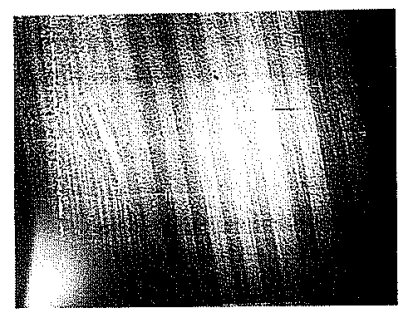

(a) Stress free, Demagnetized

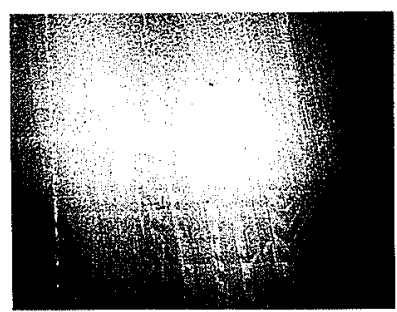

(c) Stress free, Magnetized(D.C.) $\mathrm{B}=12 \mathrm{KG}$

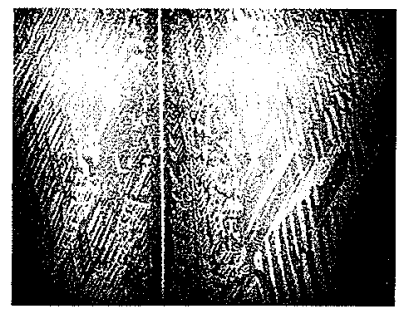

(b) Stress free, Magnetized(D.C.) $B=9 K G$

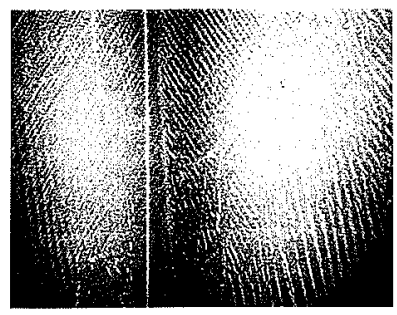

(d) $2.5 \mathrm{MPa}$ Compressive stress, Magnetized(D.C.) $B=11 \mathrm{KG}$

$1 \mathrm{~mm}$

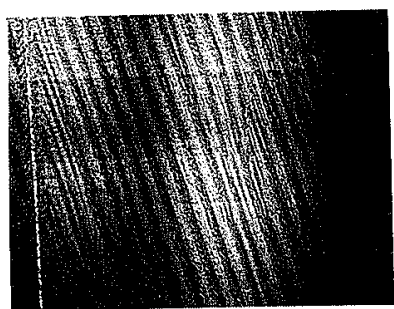

(b) $7 \mathrm{MPa}$ Compressive stress, Demagnetized

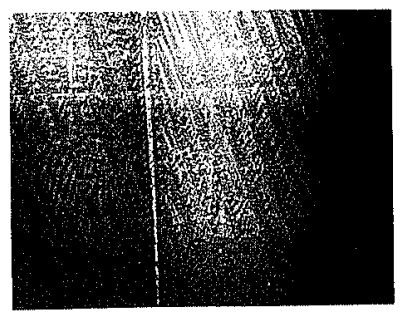

(d) $10 \mathrm{MPa}$ Compressive stress, Magnetized(D.C.) $B=11 \mathrm{KG}$

$\longdiv { \mathrm { mm } }$

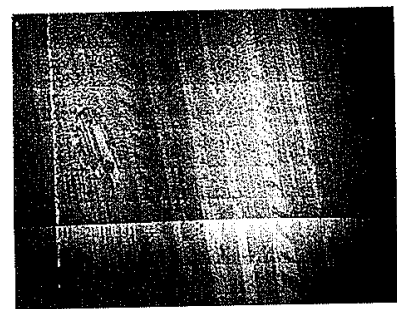

(b) Stress free, Magnetized(D.C.) $\mathrm{B}=11 \mathrm{KG}$
Fig. 8.

Magnetic domain structures in $\phi=45^{\circ}$ sample B with $\theta=45^{\circ}$ under various stress and magnetic field conditions.
Fig. 9.

Magnetic domain structures in $\phi=60^{\circ}$ sample A with $\theta=52^{\circ}$ under various stress and magnetic field conditions.
Fig. 10.

Magnetic domain structures in $\phi=75^{\circ}$ sample A with $\theta=68^{\circ}$ under various stress and magnetic field conditions. 


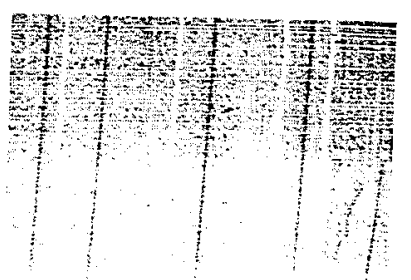

(a) Stress free, Demagnetized

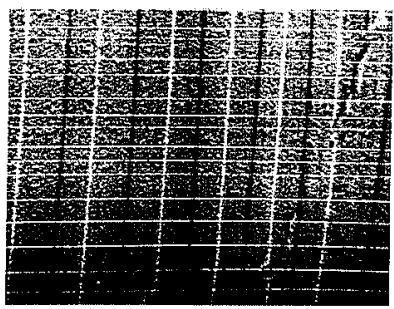

(c) $4 \mathrm{MPa}$ Compressive stress, Demagnetized

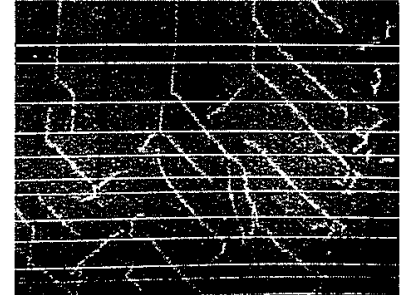

(b) Stress free, Magnetized(D.C.) $B=10 K G$

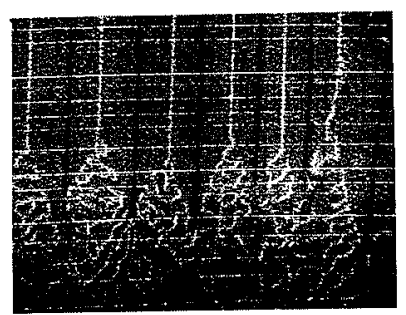

(d) $4 \mathrm{MPa}$ Compressive stress, Magnetized(D.C.) $B=10 \mathrm{KG}$
Fig. 11.

Magnetic domain structures in $\phi=90^{\circ}$ sample A with $\theta=88^{\circ}$ under various stress and magnetic field conditions.

$\overleftarrow{1 \mathrm{~mm}}$

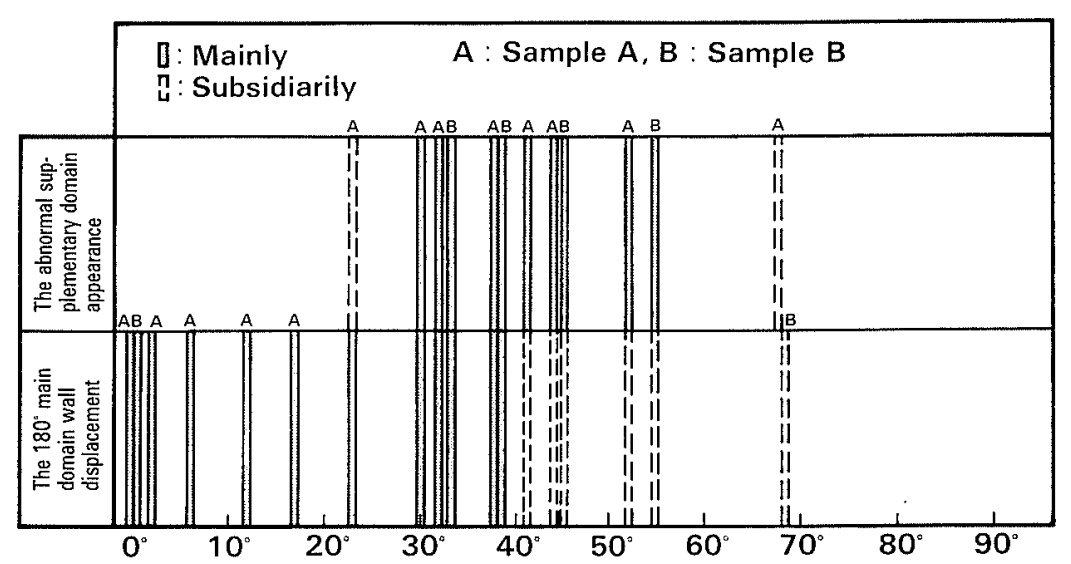

Angle between the $180^{\circ}$ main domains and magnetic field, $\theta$
Fig. 12.

A map indicating with a bar which of the $180^{\circ}$ main domain wall displacement or the abnormal supplementary domains appearance occurred mainly or subsidiarily under magnetic field in relation to angle between the $180^{\circ}$ main domains and magnetic field, $\theta$.

Table 2. Calculated formulas of strain elastic energy in the easy magnetization axes under various stress conditions of grain oriented silicon steel.

\begin{tabular}{|c|c|c|c|c|}
\hline & $x[100]$ & $y[010]$ & $z[001]$ & Reference \\
\hline 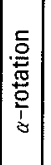 & $\begin{array}{l}\left(m^{2} a+\ell^{2} b\right)^{2} S_{11}+\left(m^{2} a+\right. \\
\left.\ell^{2} b\right)\left(\ell^{2} a+m^{2} b+c\right) S_{12}+ \\
\ell^{2} m^{2}(a-b)^{2} S_{44} \cdot \sigma^{2}\end{array}$ & $\begin{array}{l}\left(\ell^{2} a+m^{2} b+c\right)^{2} S_{11}+\left(\ell^{2} a+m^{2} b\right. \\
+c)\left\{\left(\ell^{2}+2 m^{2}\right) a+\left(2 \ell^{2}+m^{2}\right) b\right. \\
+c\} S_{12}+\left\{2 \ell^{2} m^{2}(a-b)^{2}+\left(\ell^{2} a+\right.\right. \\
\left.m^{2} b-c\right)^{2}: S_{44} \cdot \sigma^{2} / 4\end{array}$ & (same to left) & $\begin{array}{l}\ell=\sin a \\
m=\cos a\end{array}$ \\
\hline 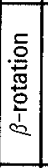 & $\begin{array}{l}\left(\mathrm{m}^{2} a+\ell^{2} c\right)^{2} S_{11}+\left(\mathrm{m}^{2} a+\right. \\
\left.\ell^{2} c\right)\left(\ell^{2} a+b+m^{2} c\right) S_{12}+ \\
\ell^{2} \mathrm{~m}^{2}(a-c)^{2} S_{44} \cdot \sigma^{2}\end{array}$ & $\begin{array}{l}\left(\ell^{2} a+b+m^{2} c\right)^{2} S_{11}+\left(\ell^{2} a+b+\right. \\
\left.m^{2} c\right)\left\{\left(\ell^{2}+2 m^{2}\right) a+b+\left(2 \ell^{2}+m^{2}\right)\right. \\
c\} S_{12}+\left\{2 \ell^{2} m^{2}(a-c)^{2}+\left(\ell^{2} a-b+\right.\right. \\
\left.\left.m^{2} c\right)^{2}\right\} S_{44} \cdot \sigma^{2} / 4\end{array}$ & (same to left) & $\begin{array}{l}\ell=\sin \beta \\
\mathrm{m}=\cos \beta\end{array}$ \\
\hline 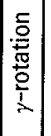 & $\begin{array}{l}a^{2} S_{11}+a(b+c)\left(\ell^{2}+m^{2}\right) \\
S_{12} \cdot \sigma^{2}\end{array}$ & $\begin{array}{l}\left(\mathrm{m}^{2} \mathrm{~b}+\ell^{2} \mathrm{c}\right)^{2} \mathrm{~S}_{11}+\left\langle\mathrm{m}^{2} \mathrm{~b}+\ell^{2} \mathrm{c}\right\rangle \\
\left(\mathrm{a}+\ell^{2} \mathrm{~b}+\mathrm{m}^{2} \mathrm{c}\right) \mathrm{S}_{12}+\ell^{2} \mathrm{~m}^{2}(\mathrm{~b}-\mathrm{c})^{2} \\
\mathrm{~S}_{44} \cdot \sigma^{2}\end{array}$ & $\begin{array}{l}\left(\ell^{2} b+m^{2} c\right)^{2} S_{11}+\left(\ell^{2} b+\right. \\
\left.m^{2} c\right)\left(a+m^{2} b+\ell^{2} c\right) S_{12} \\
+\ell^{2} m^{2}(b-c)^{2} S_{44} \cdot \sigma^{2}\end{array}$ & $\begin{array}{l}\ell=\sin (45-y) \\
m=\cos (45-;)\end{array}$ \\
\hline
\end{tabular}

Stress

b. $\sigma=$ Coating stress

a. $\sigma=$ Compressive (Tensile) stress + Coating stress

c. $\sigma=$ Residual internal tensile stress at groove
Anisotropic elastic coefficient

$S_{11}: 7.57 \times 10^{12} \mathrm{~m}^{2} / \mathrm{N}$

$S_{12}:-2.82 \times 10^{12} \mathrm{~m}^{2} / \mathrm{N}$

Deviation of Goss crystal to $X Y Z$

$\alpha$ (rotation in $X Y$ plane), $\beta(X Z), \gamma(Y Z)$ 


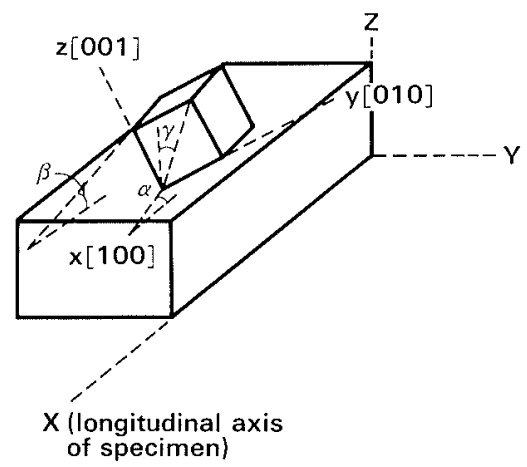

Fig. 13. Relation between coordinates $(x, y, z)$ of cubic crystal and those $(X, Y, Z)$ of specimen.
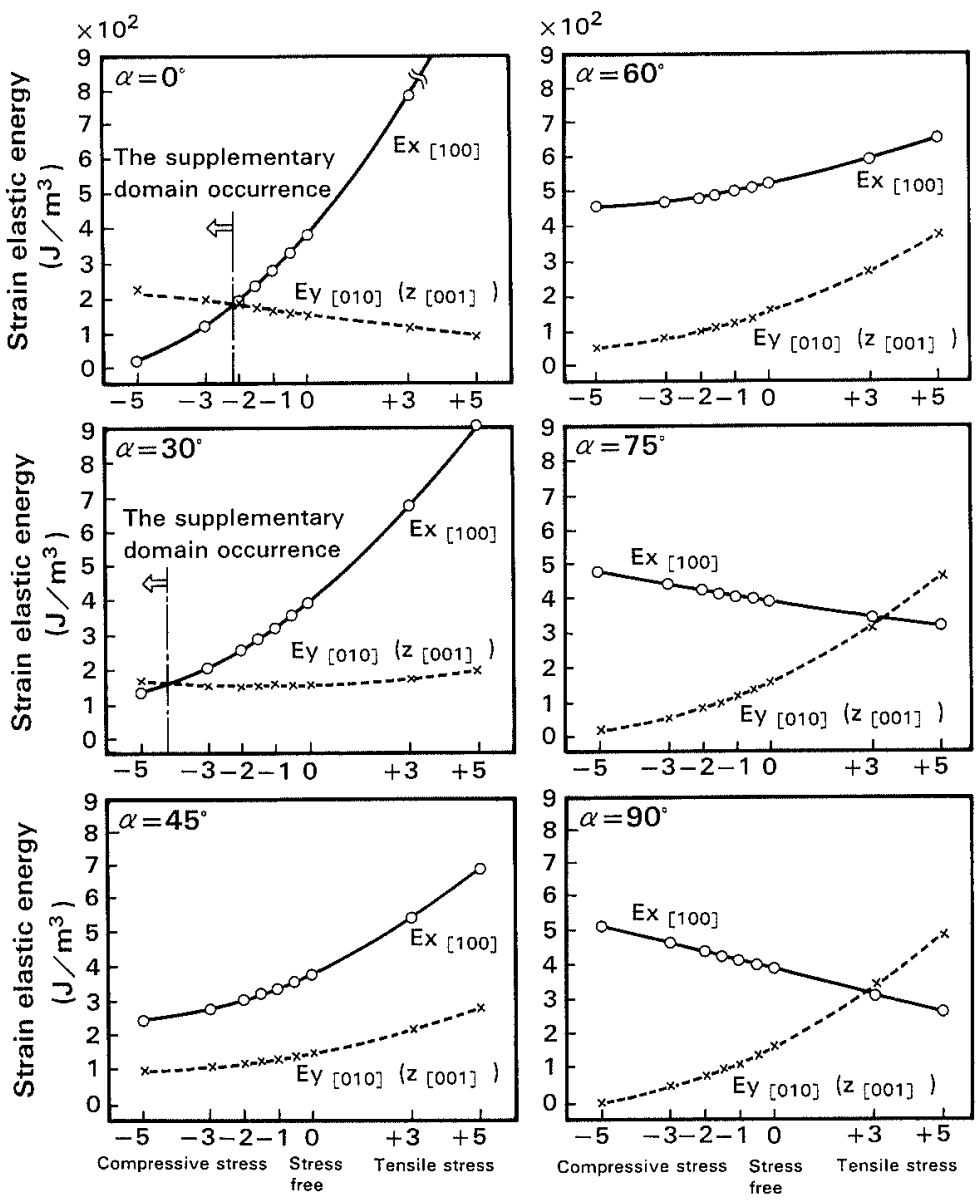

Applied stress (MPa)

Applied stress (MPa)

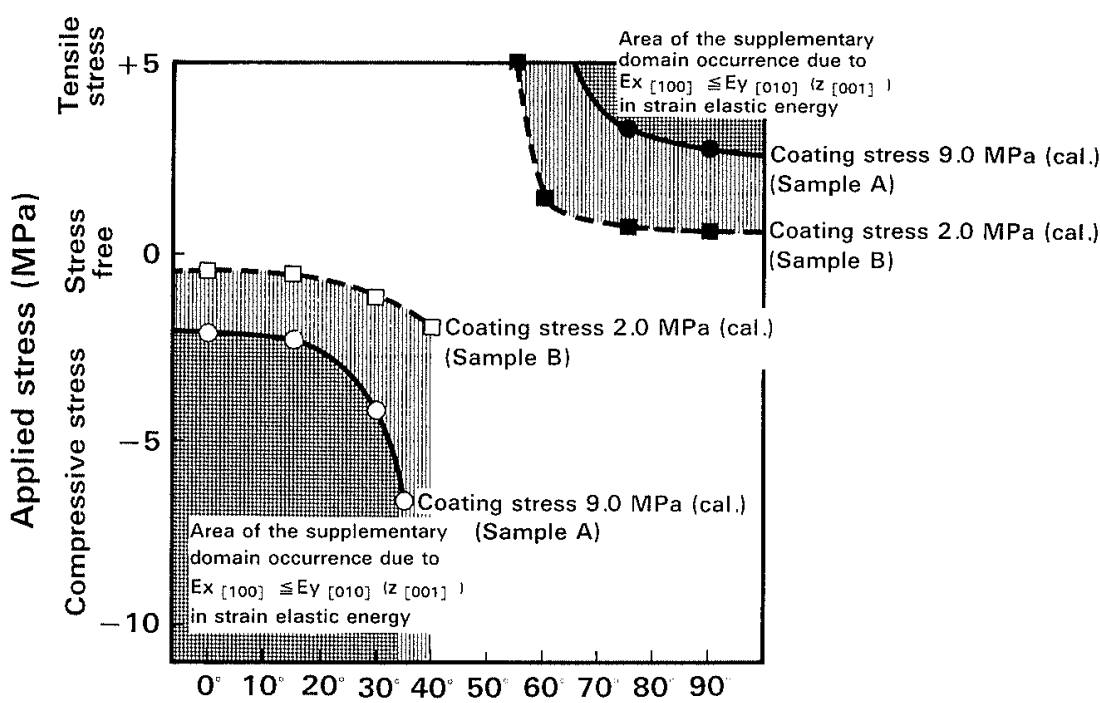

Angle between $x[100]$ direction and applied stress, $\alpha$ thickness direction. The rotation of the Goss orientation on $X Y$ plane is defined as $\alpha$ and that on $X Z$ plane as $\beta$ as well as that on $Y Z$ as $\gamma$. The calculation in this study is easily proceeded applying $\alpha$ as angle between [100] axis and applied stress. Figure 14 shows a variation of $E_{x[100]}^{\mathrm{el}}$ and $E_{y[010](z[001])}^{\mathrm{el}}$ under various applied stress conditions for sample A with every $15^{\circ}$ angles between $0^{\circ}$ and $90^{\circ}$ from $x[100]$ direction normally parallel to the rolling direction in the Goss texture. From this, it was possible to obtain Fig. 15 which indicates the occurrence region of the supplementary domains by applied stress to the samples of different coating stress in accordance with angle between $x[100]$ direction and
Fig. 14.

Influence of angle between $x[100]$ direction ( $\fallingdotseq$ the rolling direction) and applied stress direction, $\alpha$ on the strain elastic energy in easy magnetization axes $x[100], y[010]$ and $z[001]$ under various applied stress conditions in sample $\mathrm{A}$.
Fig. 15.

Chart of the supplementary domain occurrence due to $E_{x[100]} \leqq E_{y[0101}\left(E_{z[001]}\right)$ by the strain elastic energy theory depicted with a function of angle between $x$ [100] direction ( $\fallingdotseq$ the rolling direction) and applied stress direction, $\alpha$ without magnetic field. 
applied stress direction, $\alpha$. Experimental values were plotted in Fig. 16. Figure 16 proves that the strain elastic energy theory for the occurrence of the supplementary domains is available even in the different coating stress samples with considerable amount of degrees in angle between $x$ [100] direction normally parallel to the rolling direction and applied stress direction parallel to the specimen direction in this experiments. It is easily accepted $^{1,10,20)}$ that the magnetostriction in iron shows positive value when initially existed $90^{\circ}$ supplementary domains induced by stress are magnetized toward a direction parallel to the $180^{\circ}$ main domains, while it shows, on the contrary, negative value when the supplementary domains are newly generated by magnetic field. From combination of Figs. 12 and 16, it is well explained that the magnetostriction is favourably smaller negative value in the $\chi$ (angle between the magnetostriction specimen and the rolling direction) $=45^{\circ}$ sample $B$ with poor coating stress, rather than sample $A$ with large coating stress. On the other hand, in the $\chi=90^{\circ}$ sample, it is easily explained that largest positive magnetostriction value is induced by inevitable rotation of the initial $180^{\circ}$ main domains toward transverse magnetic field, which consequently brings about the singular domain structures as in Fig. 11. For the samples near the rolling direction, there was no indication of the abnormal supplementary domains by magnetic field. This may be the reason why applied stress in combination of coating stress is exclusively dominant to the magnetostriction in the rolling direction, as was illustrated by the strain elastic energy theory. ${ }^{1)}$ In fact, in the $\chi=0^{\circ}$ samples of different coating stress, the magnetostriction appeared in a similar manner to the previous report ${ }^{1)}$ as in Fig. 2 and was explained by the strain elastic energy theory as in Fig. 16. The critical angle range where the abnormal supplementary domains appear under the magnetic field, is nearly $30^{\circ}-60^{\circ}$ from the rolling direction as shown in Fig. 12. This may be explained as follows. In the magnetic field, as a matter of course, such magnetic domain

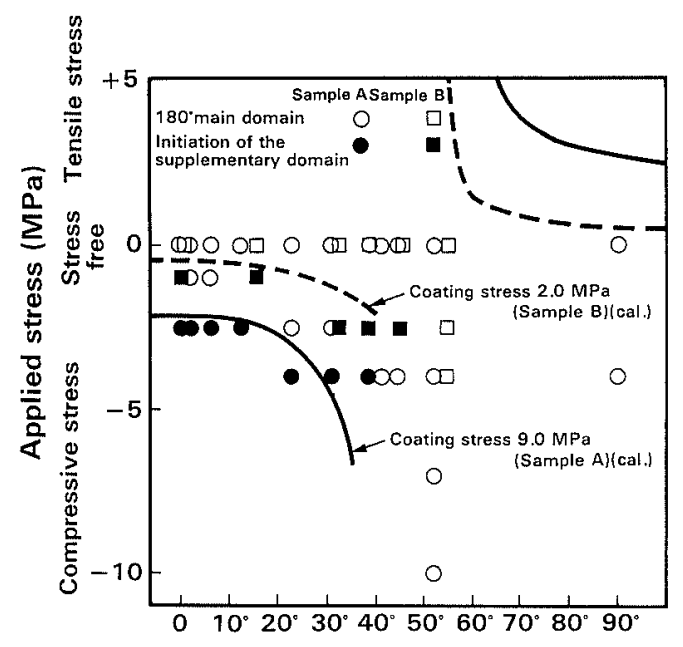

Angle between $\mathrm{x}[100]$ direction and applied stress, $\alpha$

Fig. 16. Comparison between experimental results and the calculated chart in Fig. 15 for the occurrence of the supplementary domains in accordance with angle between $x[100]$ direction ( $\fallingdotseq$ the rolling direction) and applied stress direction, $\alpha$ without magnetic field. energies as magnetic anisotropy energy and magnetostatic energy, can never be neglected. The magnetic anisotropy energy under magnetic field in the specimens with angles to the easy magnetization axes, is expressed as introduced by Eq. (1), ${ }^{10-21)}$ which is reduced to Eq. (2) by the authors for the present case.

$$
\begin{aligned}
& E_{\mathrm{k}}=K_{1}\left(\alpha_{1}^{2} \alpha_{2}^{2}+\alpha_{2}^{2} \alpha_{3}^{2}+\alpha_{3}^{2} \alpha_{1}^{2}\right) \ldots . \\
& E_{\mathrm{k}}=K_{1} \cdot(1 / 4) \sin ^{2} \theta\left(4-3 \sin ^{2} \theta\right)
\end{aligned}
$$

Introduction of Eq. (2) which is obtained by substitution of the direction cosine $\alpha_{1}([100]), \alpha_{2}([010])$ and $\alpha_{3}([001])$ in the expression (1), should be explained further in details. The magnetic anisotropy energy in specimen with $\theta$ (angle between the $180^{\circ}$ main domains and magnetic field, which means also the angle between easy magnetization axis [100] and direction of specimen parallel to magnetic field), is given in such a manner as substitution of the direction cosine to $[100] ; \alpha_{1}=\cos \theta$, that to $[010] ; \alpha_{2}=(1 / \sqrt{2}) \sin \theta$ and that to $[001]$; $\alpha_{3}=-(1 / \sqrt{2}) \sin \theta$ into Eq. (1) as follows.

$$
\begin{aligned}
E_{\mathrm{k}}= & K_{1}\left(\alpha_{1}^{2} \alpha_{2}^{2}+\alpha_{2}^{2} \alpha_{3}^{2}+\alpha_{3}^{2} \alpha_{1}^{2}\right) \\
= & K_{1}\left\{\cos ^{2} \theta \cdot(1 / 2) \sin ^{2} \theta+(1 / 2) \sin ^{2} \theta\right. \\
& \left.\quad \cdot(1 / 2) \sin ^{2} \theta+(1 / 2) \cdot \sin ^{2} \theta \cos ^{2} \theta\right\} \\
= & K_{1} \cdot(1 / 4) \sin ^{2} \theta\left(4-3 \sin ^{2} \theta\right)
\end{aligned}
$$

On the other hand, the magnetostatic energy is the sum of the potential energy as described in Eq. (3) and the spontaneous magnetization energy in Eq.(4). ${ }^{10-21)}$

$$
\begin{aligned}
& E_{\mathrm{S} 1}=-I H \cos \alpha \\
& E_{\mathrm{S} 2}=(1 / 2) N_{\mathrm{D}}\left(\Delta I_{n}\right)^{2} / \mu_{0}=(1 / 2) N_{\mathrm{D}}\left(I_{\mathrm{s}} \cos \alpha\right)^{2} / \mu_{0}
\end{aligned}
$$

Here, $I$ : Intensity of magnetization (Tesla), $H$ : Magnetic field $(\mathrm{A} / \mathrm{m}), N_{\mathrm{D}}$ : Demagnetizing factor $(\fallingdotseq 0.05), \mu_{0}$ : Initial

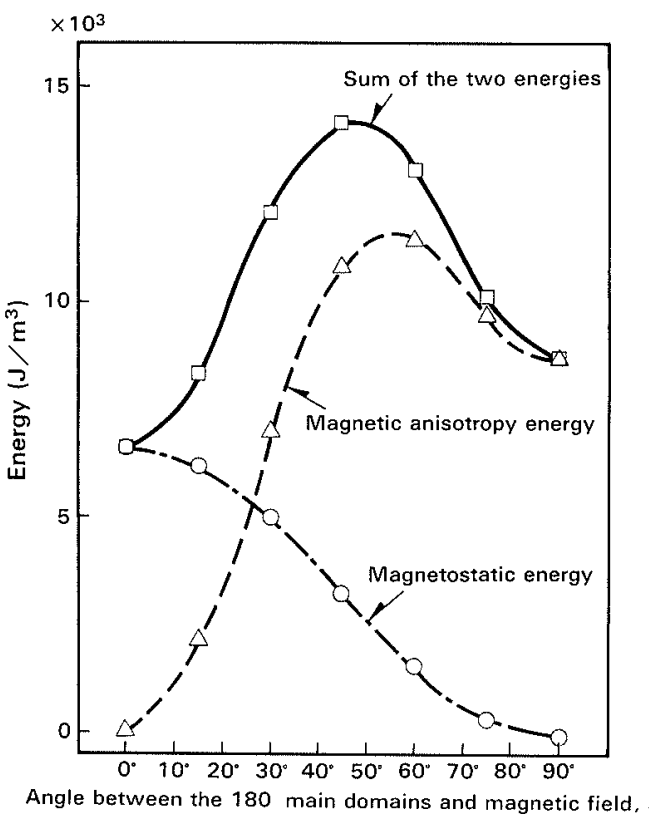

Fig. 17. The calculation results of the magnetic anisotropy energy, the magnetostatic energy and consequently, the sum of the two, in accordance with angle between the $180^{\circ}$ main domains and magnetic field, $\theta$ based on the assumption that the abnormal supplementary domains come out from the $180^{\circ}$ main domains. 
permeability $\left(\mathrm{N} / \mathrm{A}^{2}\right), \Delta I_{\mathrm{n}}$ : Spontaneous magnetization component in the direction with angle $\theta$ and $I_{\mathrm{s}}$ : Spontaneous magnetization parallel to the rolling direction $\left(I_{\mathrm{s}}^{2} / \mu_{0} \fallingdotseq 28 \times 10^{4} \mathrm{~J} / \mathrm{m}^{3}\right) .^{10,11,18,20)}$ The domain wall energy was too small compared with these energies and could be neglected. Figure $\mathbf{1 7}$ shows calculation results of the magnetic anisotropy energy, the magnetostatic energy and consequently, the sum of the two. It seems that the sum of the two energies has mountainous energy curve with a peak near about $\theta \fallingdotseq 45^{\circ}$ and that it takes nearly the same values at $\theta=30^{\circ}$ and $60^{\circ}$. As shown in Figs. 7-10, the abnormal supplementary domains seem to appear mainly from the $180^{\circ}$ main domains when they are exposed to magnetic field. The most distinctive evidence is Fig. 7(c) where it seems that the abnormal supplementary domains come out clearly from the $180^{\circ}$ main domains and even that the $90^{\circ}$ supplementary domains which were generated by applied stress as in Fig. 7(a) still remains even after this transformation. In the case, however, where there is few $180^{\circ}$ main domains, this calculation has to be refined more precisely on sophisticated combination of magnetic domain structure. Thus, it seems reasonable that the sum of the two energies in Fig. 17 determines the appearance of the abnormal supplementary domains under magnetic field as shown in Fig. 12. The formation of the abnormal supplementary domains under magnetic field might contribute to diminish total magnetic energies, which is conspicuous for angles $\theta \fallingdotseq 30^{\circ}-60^{\circ}$, in samples which had initially the main $180^{\circ}$ domains with angle $\theta$ to magnetic field. Furthermore, it should be referred that the strain elastic energy, which is efficiently dominant on magnetostriction in the rolling direction by the formation of the supplementary $90^{\circ}$ domains by stresses, has rather small values compared with the sum of the two energies in Fig. 17, and thus, it is reasonable that the abnormal supplementary domains under magnetic field appear irrelevantly of the magnitude of applied compressive stress and even of coating stress in the specimen with $\theta \fallingdotseq 30^{\circ}-60^{\circ}$ as shown in Figs. 7-10.

\section{Conclusion}

The magnetostriction of grain oriented silicon steel sheets with angle such as $45^{\circ}$ or $90^{\circ}$ off the rolling direction which recently became noticeable on the point of noise at joint portion of limb and yoke in transformer, was investigated. Research was carried out both on the magnetostriction and an observation of magnetic domains in changing direction of grain oriented silicon steel sheet. It was made clear that the strain elastic energy theory for the occurrence of the $90^{\circ}$ supplementary domains by applied stress without magnetic field holds even in samples of different angle from the rolling direction. In sample with $45^{\circ}$ direction off the rolling direction, the magnetostriction indicated negative value regardless of compressive stress applied to the specimen and showed favourably smaller negative value in poor coating stress sample rather than in good one. This was explained by a fact that the $90^{\circ}$ supplementary domains by compressive applied stress by the theory, which cause positive magnetostriction when the specimen is exposed to magnetic field, is compensated by appearance of the abnormal supplementary domains newly generated under magnetic field, which causes negative magnetostriction. The abnormal supplementary domains occur in specimen with the angle to the rolling direction of about $30^{\circ}-60^{\circ}$, and this was tried to explain by magnetic domain energy. In sample with $90^{\circ}$ direction off the rolling direction, the magnetostriction indicated the largest positive value which was easily supposed by singular domains occurred when the main $180^{\circ}$ domains are exposed to magnetic field transversely.

\section{REFERENCES}

1) H. Masui, M. Mizokami, Y. Matsuo and H. Mogi: ISIJ Int., 35 (1995), 409.

2) A. J. Moses: IEEE Trans. Magn., MAG, 15 (1979), 1575.

3) A. J. Moses and P. S. Phillips: IEEE Trans. Magn, $M A G, 14$ (1978), 353.

4) S. M. Pegler: AIP Conf. Proc., 24 (1974), 718.

5) W. D. Corner and J. J. Mason: Brit. J. Appl. Phys., 15 (1964), 709.

6) T. Yamamoto, S. Taguchi, A. Sakakura and T. Nozawa: IEEE Trans., $M A G, 8$ (1972), 677.

7) A. Basak and C. R. G. Higgs: IEEE Trans. Magn., MAG, 18 (1982), 670 .

8) Z. Valkovic: Phys. Scr., T24 (1988), 71.

9) P. Silvester and M. V. K. Chari: IEEE Trans., PAS, 89 (1970), 1642.

10) S. Chikazumi: Physics of Magnetism, John Wiley \& Sons. Inc., New York, (1964), 152.

11) S. Taguchi: The Electric Magnetic Steel (Japanese), Nippon Steel Corp., (1979), 86.

12) R. M. Bozorth: Ferromagnetism, IEEE Press, (1993), 595

13) R. Becker and W. Doering: Ferromagnetismus, Springer, Berlin, (1939), 482

14) H. J. Williams, R. M. Bozorth and W. Shockley: Phys. Rev., 75 (1949), 115

15) H. J. Williams and W. Shockley: Phys. Rev., 75 (1949), 178.

16) W. Heisenberg: Z. Phys., 49 (1928), 619.

17) L. Neel: J. Phys. Radium, 15 (1954), 225.

18) J. W. Shilling and G. L. Houze: IEEE Trans. Magn., MAG, 10 (1974), 195.

19) S. Kaya: Strong Magnetism (Japanese), Iwanami, Tokyo, (1951), 103.

20) S. Chikazumi: Physics of Ferromagnetism, Vol. II (Japanese), Shokabo, Japan, (1984), 106.

21) K. Ohta: Fundamental of Magnetism Engineering (Japanese), Kyoritsu Shuppan, Japan, (1989), 273.

22) H. Masui: IEEE Trans. Magn., MAG, 31 (1995), 930. 